\title{
Set families and Foulkes modules
}

\author{
Rowena Paget • Mark Wildon
}

Received: 14 August 2010 / Accepted: 23 March 2011 / Published online: 21 April 2011

(C) Springer Science+Business Media, LLC 2011

\begin{abstract}
We construct a new family of homomorphisms from Specht modules into Foulkes modules for the symmetric group. These homomorphisms are used to give a combinatorial description of the minimal partitions (in the dominance order) which label the irreducible characters appearing as summands of the characters of Foulkes modules. The homomorphisms are defined using certain families of subsets of the natural numbers. These families are of independent interest; we prove a number of combinatorial results concerning them.
\end{abstract}

Keywords Foulkes' conjecture $\cdot$ Specht module $\cdot$ Foulkes module $\cdot$ Module homomorphism $\cdot$ Closed set family

\section{Introduction}

The aim of this paper is to construct a new family of homomorphisms from Specht modules into Foulkes modules, and to explore some of the consequences for the structure of Foulkes modules. Foulkes modules are the object of the longstanding Foulkes' Conjecture, first made at the end of Sect. 1 of [4], which spans representation theory, invariant theory and combinatorics. We shall discuss some of these connections in this introduction.

\footnotetext{
R. Paget

School of Mathematics, Statistics and Actuarial Science, University of Kent, Cornwallis Building, Canterbury, Kent CT2 7NF, UK

e-mail: r.e.paget@kent.ac.uk

M. Wildon $(\bowtie)$

Department of Mathematics, Royal Holloway, University of London, Egham Hill, Egham TW20 0EX, UK

e-mail: mark.wildon@rhul.ac.uk
} 
Let $S_{r}$ denote the symmetric group of degree $r$. For $m, n \in \mathbf{N}$, the Foulkes module $H^{\left(m^{n}\right)}$ is defined to be the permutation module for $\mathbf{Z} S_{m n}$ given by the action of the symmetric group $S_{m n}$ on the collection of set partitions of a set of size $m n$ into $n$ sets each of size $m$. Let $\phi^{\left(m^{n}\right)}$ be the permutation character afforded by $H^{\left(m^{n}\right)}$. We shall refer to these characters as Foulkes characters. Foulkes' Conjecture asserts that if $m, n$ are natural numbers with $m<n$, and $\chi$ is an irreducible character of $S_{m n}$, then the multiplicity of $\chi$ in $\phi^{\left(m^{n}\right)}$ is at least as great as the multiplicity of $\chi$ in $\phi^{\left(n^{m}\right)}$.

Foulkes' Conjecture can be recast for GL( $V)$-modules, where $V$ is a finite dimensional complex vector space. Put in these terms, it claims that if $m<n$ then there is an embedding of GL( $V)$-modules $S^{n}\left(S^{m} V\right) \hookrightarrow S^{m}\left(S^{n} V\right)$, where $S^{m}$ denotes the $m$ th symmetric power. In [1, page 352], Brion used this interpretation and ideas from geometric invariant theory to show that Foulkes' Conjecture is true provided $n$ is large compared to $m$.

Finally, we mention that taking formal characters of $\mathrm{GL}(V)$-modules gives a purely combinatorial formulation of Foulkes' Conjecture in terms of symmetric functions. In this setting it states that if $m<n$ then the difference of plethysms of Schur functions $s_{n}\left[s_{m}\right]-s_{m}\left[s_{n}\right]$ is a sum of Schur functions with non-negative coefficients. Understanding these plethysm coefficients was identified by Stanley in [10, Problem 9] as an important open positivity problem in algebraic combinatorics.

For a full outline of the results proved on Foulkes characters in this article see Sect. 2 below. Our main result (see Theorem 2) gives a combinatorial description, in terms of certain set families, of the minimal partitions which label irreducible characters appearing as summands of Foulkes characters. (Here minimality is with respect to the dominance order on partitions.) This theorem gives the strongest general results on Foulkes characters known to date.

Using Theorem 2, the authors have found all minimal constituents of the Foulkes characters $\phi^{\left(m^{n}\right)}$ for $m+n \leq 20$. The data, together with the associated computer software, are available from the second author's website: www.ma.rhul.ac.uk/ uvah099.

It is an important feature of our approach that Theorem 2 is proved using explicitly defined maps between integral Specht and Foulkes modules for the symmetric group. This 'characteristic-free' approach is well-suited to our results, and does not create any significant extra difficulties in their proofs. For background on integral modules the reader is referred to [2, Chap. 11]. A subsequent paper by the authors will apply the results herein to study the behaviour of Foulkes modules over fields of prime characteristic.

Although Foulkes' Conjecture remains open, some progress has been made. Besides the asymptotic result of Brion already mentioned, the conjecture is known to hold when $m \leq 4$. When Foulkes made his conjecture in 1950 it was already known to hold by the work of Thrall (see [11, Theorems III and IV]) in the case $m=2$. It was proved when $m=3$ by Dent and Siemons [3]. The most recent progress was made in 2008 by McKay [8], who proved it when $m=3$ and $m=4$. McKay's proof uses a family of maps $\psi_{\left(n^{m}\right)}: H^{\left(n^{m}\right)} \rightarrow H^{\left(m^{n}\right)}$ which were first defined by Wagner and Siemons ${ }^{1}$ in 1986. McKay's main result is that if $\psi_{\left(m^{m}\right)}$ is invertible then $\psi_{\left(n^{m}\right)}$

\footnotetext{
${ }^{1}$ See [8]. These maps were independently defined by Stanley: see the discussion following Problem 9 in [10]. Both Wagner and Siemons, and Stanley formulated stronger versions of Foulkes' Conjecture (and
} 
is injective for any $n>m$. The maps $\psi_{\left(m^{m}\right)}$ for $m \leq 4$ were already known to be invertible by the work of Müller and Neunhöffer [9], so Foulkes' Conjecture is proved in these cases.

The main contribution of [9] was to show that $\psi_{\left(5^{5}\right)}$ has a non-zero kernel. It is however still possible that the maps $\psi_{\left(n^{m}\right)}$ will have a role in proving or disproving Foulkes' Conjecture. In particular, a conjecture of Johannes Siemons ${ }^{2}$ implies that if the kernel of $\psi_{\left(n^{m}\right)}$ is non-zero, then the ordinary character of $\operatorname{ker} \psi_{\left(n^{m}\right)}$ has one of the minimal constituents of $\phi^{\left(n^{m}\right)}$ classified in our Theorem 2 as a summand. It seems likely that any counterexample to Foulkes' Conjecture will involve one of these minimal constituents.

\section{Outline}

Our homomorphisms are defined using certain families of subsets of the natural numbers. We shall need the following combinatorial definitions.

Definition 1 Let $X=\left\{x_{1}, \ldots, x_{m}\right\}$ and $Y=\left\{y_{1}, \ldots, y_{m}\right\}$ be subsets of the natural numbers, written so that $x_{1}<x_{2}<\cdots<x_{m}$ and $y_{1}<y_{2}<\cdots<y_{m}$. We say that $Y$ majorizes $X$, and write $X \preceq Y$, if $x_{i} \leq y_{i}$ for each $i$.

The reader may find it helpful to refer to Fig. 2 in Sect. 6.2 below, which shows part of the lattice of 4 -subsets of $\mathbf{N}$ under the majorization order.

Definition 2 A set family of shape $\left(m^{n}\right)$ is a collection of $n$ distinct $m$-subsets of the natural numbers. A set family $\mathcal{P}$ is closed if whenever $Y \in \mathcal{P}$ and $X \prec Y$, then $X \in \mathcal{P}$.

In the following definition, $\lambda^{\prime}$ denotes the conjugate of the partition $\lambda$.

Definition 3 Let $\lambda$ be a partition with largest part of size $a$. A set family has type $\lambda$ if it has exactly $\lambda_{i}^{\prime}$ sets containing $i$ for each $i \in\{1, \ldots, a\}$.

It is easily seen that if $\mathcal{P}$ is a closed set family then for any $i \in \mathbf{N}$, at least as many sets in $\mathcal{P}$ contain $i$ as contain $i+1$. Closed set families therefore have well-defined types. The reason for working with conjugate partitions will be seen in Theorem 1 below.

Recall that if $\lambda$ and $\mu$ are partitions of $n$ then we say that $\lambda$ dominates $\mu$, and write $\lambda \unrhd \mu$, if $\sum_{i=1}^{j} \lambda_{i} \geq \sum_{i=1}^{j} \mu_{i}$ for all $j \in \mathbf{N}$. (If $i$ exceeds the number of parts of $\lambda$ or $\mu$, then the corresponding part should be taken to be 0 .)

Definition 4 Let $\mathcal{P}$ be a set family of shape $\left(m^{n}\right)$ and type $\lambda$. We say that $\mathcal{P}$ is minimal if there is no set family $\mathcal{Q}$ of shape $\left(m^{n}\right)$ and type $\mu$ with $\mu \triangleleft \lambda$.

also of Howe's Conjecture on GL( $V)$-modules; see [6, Sect. 2.5]) using these maps. A counterexample to these stronger conjectures is given in [9].

${ }^{2}$ Seminar given at Castro Urdiales, October 2007. 
It is an important fact that minimal set families are always closed; we shall prove this fact when we first need it, in Proposition 3 in Sect. 5.2 below.

Finally, if $\lambda$ is a partition of $r$, we denote by $S^{\lambda}$ the associated Specht module for $\mathbf{Z} S_{r}$. The reader is referred to Sect. 3 of this paper for the definition of Specht modules and our notational conventions for Foulkes modules.

We are now ready to state our main results. Let $m$ be odd. In Sect. 3.2 we define for each closed set family $\mathcal{P}$ of shape $\left(\mathrm{m}^{n}\right)$ and type $\lambda$, a homomorphism $f_{\mathcal{P}}: S^{\lambda} \rightarrow H^{\left(m^{n}\right)}$. A critical result, which we prove in Sect. 4 below, is that these homomorphisms are well-defined.

Theorem 1 Let $m$ be odd and let $n \in \mathbf{N}$. Let $\mathcal{P}$ be a closed set family of shape $\left(m^{n}\right)$ and type $\lambda$. The map $f_{\mathcal{P}}: S^{\lambda} \rightarrow H^{\left(m^{n}\right)}$ defined in Sect. 3.2 is a well-defined injective homomorphism from $S^{\lambda}$ to $H^{\left(m^{n}\right)}$.

Let $\chi^{\lambda}$ be the irreducible character afforded by the Specht module $S^{\lambda}$. (More precisely, if $\lambda$ is a partition of $r$, then $\chi^{\lambda}$ is the character of the $\mathbf{Q} S_{r}$-module $S^{\lambda} \otimes_{\mathbf{Z}} \mathbf{Q}$.) It is well known that every irreducible character of a symmetric group is equal to a $\chi^{\lambda}$ : see, for instance, [7, Theorem 4.12]. In terms of characters, Theorem 1 states that if there is a closed set family of type $\left(m^{n}\right)$ and type $\lambda$ then $\left\langle\phi^{\left(m^{n}\right)}, \chi^{\lambda}\right\rangle \geq 1$.

If $\pi$ is a character of $S_{r}$ and $\lambda$ is a partition of $r$, then we shall say that $\chi^{\lambda}$ is a minimal constituent of $\pi$ if $\left\langle\pi, \chi^{\lambda}\right\rangle \geq 1$ and $\left\langle\pi, \chi^{\mu}\right\rangle=0$ if $\mu \triangleleft \lambda$. In Sect. 5 we prove the following theorem which characterises minimal constituents of Foulkes characters.

Theorem 2 Let $m, n \in \mathbf{N}$.

(i) If $m$ is even then the unique minimal constituent of $\phi^{\left(m^{n}\right)}$ is $\chi^{\left(m^{n}\right)}$.

(ii) If $m$ is odd then $\chi^{\lambda}$ is a minimal constituent of $\phi^{\left(m^{n}\right)}$ if and only if there is a minimal set family of shape $\left(\mathrm{m}^{n}\right)$ and type $\lambda$.

We also show that if $m$ is even then $\left\langle\phi^{\left(m^{n}\right)}, \chi^{\left(m^{n}\right)}\right\rangle=1$, and that part (ii) of the above theorem can be sharpened as follows.

Theorem 3 Let $m$ be odd and let $n \in \mathbf{N}$. Suppose that $\chi^{\lambda}$ is a minimal constituent of $\phi^{\left(m^{n}\right)}$. If $\mathcal{P}_{1}, \ldots, \mathcal{P}_{d}$ are the set families of shape $\left(m^{n}\right)$ and type $\lambda$, then $\mathcal{P}_{1}, \ldots, \mathcal{P}_{d}$ are closed, and the homomorphisms $f_{\mathcal{P}_{1}}, \ldots, f_{\mathcal{P}_{d}}$ are a $\mathbf{Z}$-basis for $\operatorname{Hom}_{\mathbf{Z} S_{m n}}\left(S^{\lambda}, H^{\left(m^{n}\right)}\right)$. In particular, $\left\langle\phi^{\left(m^{n}\right)}, \chi^{\lambda}\right\rangle=d$.

We pause to give a small example that will illustrate these theorems. We take $m=3$ and $n=4$. The three closed set families of shape $\left(3^{4}\right)$ are

$$
\begin{aligned}
& \{\{1,2,3\},\{1,2,4\},\{1,2,5\},\{1,2,6\}\}, \\
& \{\{1,2,3\},\{1,2,4\},\{1,2,5\},\{1,3,4\}\}, \\
& \{\{1,2,3\},\{1,2,4\},\{1,3,4\},\{2,3,4\}\},
\end{aligned}
$$


of types $(6,2,2,2),(5,4,2,1)$ and $(4,4,4)$, respectively. Since these partitions are incomparable in the dominance order, and (by Proposition 3 in Sect. 5.2) any minimal set family is closed, the set families above are minimal. It now follows from Theorems 2 and 3 that $\phi^{\left(3^{4}\right)}$ has $\chi^{(6,2,2,2)}, \chi^{(5,4,2,1)}$ and $\chi^{(4,4,4)}$ as summands, each with multiplicity 1 . Moreover, if $\left\langle\phi^{\left(3^{4}\right)}, \chi^{\mu}\right\rangle \geq 1$ then $\mu$ dominates one of these partitions. The presence of such larger constituents cannot be detected by the homomorphisms $f_{\mathcal{P}}$.

Small examples of this kind are apt to create the false impression that every closed set family is minimal and is the unique set family of its type. In Sect. 6.1 we prove the following theorem which clarifies the relationship between these properties.

Theorem 4 If $\mathcal{P}$ is the unique set family of its type, then $\mathcal{P}$ is minimal. If $\mathcal{P}$ is a minimal set family then $\mathcal{P}$ is closed. There exist closed set families that are not minimal, and minimal set families that are not unique for their type.

The existence of minimal set families that are not unique for their type is of particular significance, since such families demonstrate that the multiplicity $d$ in Theorem 3 can be strictly greater than 1 .

Even with the help of Theorem 2, it appears to be a difficult matter to decide, when $m$ is odd, whether a given partition of $m n$ labels a minimal constituent of the Foulkes character $\phi^{\left(m^{n}\right)}$. In Sect. 6.2 we give a construction that gives some of these partitions. We prove that this construction gives every such partition if and only if $n \leq 5$.

We end in Sect. 7 by defining the generalised Foulkes characters $\phi^{\mu}$ considered in [8] and showing how to obtain their minimal constituents from the minimal constituents of the $\phi^{\left(m^{n}\right)}$. This section may be read independently of the rest of the paper.

\section{Specht modules and homomorphisms}

In this section we recall the definition of Specht modules as submodules of Young permutation modules and define the homomorphisms $f_{\mathcal{P}}$.

The following notation simplifies these definitions and will be found very useful in the proofs which follow. Given a partition $\lambda$ of $r$ with largest part of size $a$, let $A(\lambda)$ be the set consisting of the symbols $i_{j}$ for $1 \leq i \leq a$ and $1 \leq j \leq \lambda_{i}^{\prime}$. We say that $i$ is the number and $j$ is the index of the symbol $i_{j}$.

\subsection{Specht modules}

Let $\lambda$ be a partition of $r$. A $\lambda$-tableau is an assignment of the elements of $A(\lambda)$ to the boxes of the Young diagram of $\lambda$. Given a $\lambda$-tableau $t$, we obtain the associated tabloid $\mathbf{t}$ by disregarding the order of the elements within the rows of $t$. For example, if

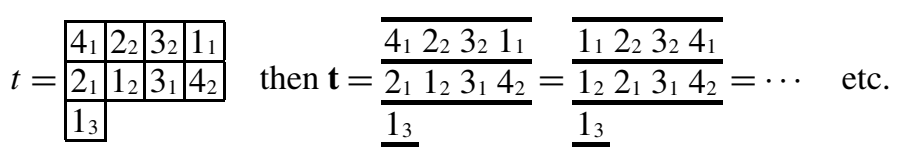


We may identify $S_{r}$ with the symmetric group on $A(\lambda)$. After this identification is made, the natural permutation action of $S_{r}$ on the set of $\lambda$-tableaux gives rise to a well-defined action of $S_{r}$ on the set of $\lambda$-tabloids. We denote the associated permutation module for $\mathbf{Z} S_{r}$ by $M^{\lambda}$; it is the Young permutation module corresponding to $\lambda$. For example, $M^{(r-1,1)}$ affords the natural integral representation of $S_{r}$ as $r \times r$ permutation matrices.

Given a $\lambda$-tableau $t$, we let $C(t)$ be the subgroup of $S_{r}$ consisting of those elements which fix setwise the columns of $t$. Define $b_{t} \in \mathbf{Z} S_{r}$ by

$$
b_{t}=\sum_{\tau \in C(t)} \operatorname{sgn}(\tau) \tau \text {. }
$$

The polytabloid corresponding to $t$ is the element $e_{t} \in M^{\lambda}$ defined by

$$
e_{t}=\mathbf{t} b_{t}
$$

The Specht module $S^{\lambda}$ is defined to be the submodule of $M^{\lambda}$ spanned by the $\lambda$ polytabloids. An easy calculation shows that if $\sigma \in S_{r}$ then $\left(e_{t}\right) \sigma=e_{t \sigma}$, and so $S^{\lambda}$ is cyclic, generated by any single polytabloid.

It follows from Theorem 4.12 of [7] that the rational $\mathbf{Q} S_{r}$-modules $S^{\lambda} \otimes_{\mathbf{Z}} \mathbf{Q}$ for $\lambda$ a partition of $r$ are irreducible, and that they afford all the ordinary irreducible characters of $S_{r}$.

Let $t_{\lambda}$ be the $\lambda$-tableau whose $i$ th column is $i_{1}, \ldots, i_{\lambda_{i}^{\prime}}$ when read from top to bottom. Note that the elements of $C\left(t_{\lambda}\right)$ permute the indices of symbols in $A(\lambda)$ while leaving the numbers unchanged.

\subsection{Definition of the homomorphisms $f_{\mathcal{P}}$}

Throughout this section, let $m, n \in \mathbf{N}$ and let $\lambda$ be a partition of $m n$. After identifying $S_{m n}$ with the symmetric group on the set $A(\lambda)$, the elements of the canonical permutation basis of $H^{\left(m^{n}\right)}$ are given by the following definition.

Definition 5 An indexed set partition of shape $\left(\mathrm{m}^{n}\right)$ and type $\lambda$ is a set partition of $A(\lambda)$ into $n$ sets each of size $m$.

Our notation allows us to pass easily from set families to indexed set partitions.

Definition 6 Let $\mathcal{P}$ be a set family of shape $\left(m^{n}\right)$ and type $\lambda$. Order the sets making up $\mathcal{P}$ lexicographically, so that $\mathcal{P}=\left\{X_{1}, \ldots, X_{n}\right\}$ where $X_{1}<X_{2}<\cdots<X_{n}$. The indexed set partition associated to $\mathcal{P}$ is the indexed set partition of type $\lambda$ obtained by appending indices to the elements of the sets $X_{1}, \ldots, X_{n}$ so that the elements of $X_{1}$ all get the index 1, and an element $i \in X_{r}$ is given the smallest index not appended to any $i$ appearing in $X_{1}, \ldots, X_{r-1}$.

For example, the indexed set partition associated to the closed set family

$$
\mathcal{Q}=\{\{1,2,3\},\{1,2,4\},\{1,2,5\},\{1,3,4\}\}
$$


of type $(5,4,2,1)$ is

$$
u=\left\{\left\{1_{1}, 2_{1}, 3_{1}\right\},\left\{1_{2}, 2_{2}, 4_{1}\right\},\left\{1_{3}, 2_{3}, 5_{1}\right\},\left\{1_{4}, 3_{2}, 4_{2}\right\}\right\} \in H^{\left(3^{4}\right)} .
$$

Since $S^{\lambda}$ is generated by the polytabloid $e_{t_{\lambda}}$, any homomorphism from $S^{\lambda}$ is determined by its effect on $e_{t_{\lambda}}$. Specifically, if $f: S^{\lambda} \rightarrow M$ is a homomorphism of $S_{m n}$-modules, $t$ is a $\lambda$-tableau and $\sigma \in S_{m n}$ is such that $t_{\lambda} \sigma=t$, then $e_{t} f=\left(e_{t_{\lambda}} f\right) \sigma$.

Definition 7 Let $\mathcal{P}$ be a closed set family of shape $\left(m^{n}\right)$ and type $\lambda$ where $m$ is odd. We define $f_{\mathcal{P}}: S^{\lambda} \rightarrow H^{\left(m^{n}\right)}$ by $e_{t_{\lambda}} f_{\mathcal{P}}=u b_{t_{\lambda}}$ where $u$ is the indexed set partition associated to $\mathcal{P}$.

If $\mathcal{Q}$ is as above then the homomorphism $f_{\mathcal{Q}}: S^{(5,4,2,1)} \rightarrow H^{\left(3^{4}\right)}$ is defined on the generator $e_{t_{(5,4,2,1)}}$ of $S^{(5,4,2,1)}$ by

$$
e_{t_{(5,4,2,1)}} \mapsto\left\{\left\{1_{1}, 2_{1}, 3_{1}\right\},\left\{1_{2}, 2_{2}, 4_{1}\right\},\left\{1_{3}, 2_{3}, 5_{1}\right\},\left\{1_{4}, 3_{2}, 4_{2}\right\}\right\} b_{t_{(5,4,2,1)}} .
$$

We remark that while we have, for definiteness, given an explicit scheme for passing from set families to indexed set partitions, a different choice will at most lead to changes of sign in the maps $f_{\mathcal{P}}$. For example, if in our index appending scheme, the lexicographic order on sets is replaced with the colexicographic order, then the homomorphism above would instead be defined by

$$
\begin{aligned}
e_{t_{(5,4,2,1)}} & \mapsto\left\{\left\{1_{1}, 2_{1}, 3_{1}\right\},\left\{1_{2}, 2_{2}, 4_{1}\right\},\left\{1_{3}, 3_{2}, 4_{2}\right\},\left\{1_{4}, 2_{3}, 5_{1}\right\}\right\} b_{t_{(5,4,2,1)}} \\
& =-\left\{\left\{1_{1}, 2_{1}, 3_{1}\right\},\left\{1_{2}, 2_{2}, 4_{1}\right\},\left\{1_{3}, 2_{3}, 5_{1}\right\},\left\{1_{4}, 3_{2}, 4_{2}\right\}\right\} b_{t_{(5,4,2,1)}}
\end{aligned}
$$

\section{Proof of Theorem 1}

For technical reasons it will be useful to deal with the case $m=1$ separately. The only closed set family of shape $\left(1^{n}\right)$ is $\mathcal{P}=\{\{1\},\{2\}, \ldots,\{n\}\}$, which has type $(n)$. The homomorphism $f_{\mathcal{P}}: S^{(n)} \rightarrow H^{\left(1^{n}\right)}$ is defined by

$$
e_{t_{(n)}} \mapsto\left\{\left\{1_{1}\right\},\left\{2_{1}\right\}, \ldots,\left\{n_{1}\right\}\right\} .
$$

Since $S^{(n)}$ is the trivial $\mathbf{Z} S_{n}$-module, this map is clearly well-defined and injective.

To show that the homomorphisms $f_{\mathcal{P}}$ are well-defined when $m \geq 3$, we shall use the description of the Specht module $S^{\lambda}$ given by Garnir relations. The following lemma states a suitable form of these relations in our numbers-and-indices notation.

Lemma 1 Let $U$ be a $\mathbf{Z}$-free $\mathbf{Z} S_{r}$-module, let $\lambda$ be a partition of $r$ and let $t=t_{\lambda}$. If $u \in U$ is such that

$$
u b_{t} \sum_{\sigma \in S_{X \cup Y}} \sigma \operatorname{sgn}(\sigma)=0
$$

for every pair of subsets

$$
X \subseteq\left\{i_{j}: 1 \leq j \leq \lambda_{i}^{\prime}\right\}
$$




$$
Y \subseteq\left\{(i+1)_{j}: 1 \leq j \leq \lambda_{i+1}^{\prime}\right\}
$$

such that $|X|+|Y|>\lambda_{i}^{\prime}$, then there is a homomorphism of $\mathbf{Z} S_{r}$-modules $f: S^{\lambda} \rightarrow U$ such that $e_{t} f=u b_{t}$.

Proof It follows from the remark at the top of page 102 of [5] that the kernel of the surjective map $\mathbf{Z} S_{r} \rightarrow S^{\lambda}$ defined by $x \mapsto e_{t} x$ is generated, as a right $\mathbf{Z} S_{r}$-ideal, by elements of the following two types:

- $1-\left(i_{j}, i_{k}\right)$ for $i_{j}, i_{k} \in A(\lambda)$.

- $G_{X, Y}=\sum \sigma \operatorname{sgn}(\sigma)$, where $X$ and $Y$ are as in the statement of the lemma and the sum is over a set of right-coset representatives for the cosets of $S_{X} \times S_{Y}$ in $S_{X \cup Y}$.

Clearly $u b_{t}$ is killed by elements of the first type, so to prove the lemma, it will suffice to show that $u b_{t} G_{X, Y}=0$ for each $G_{X, Y}$. As in the proof of Theorem 7.2 in [7], we set $\bar{S}_{Z}=\sum_{\sigma \in Z} \sigma \operatorname{sgn}(\sigma)$ for a subset $Z$ of $S_{r}$. Note that $\bar{S}_{X} \bar{S}_{Y} G_{X, Y}=$ $\bar{S}_{X \cup Y}$. By hypothesis $u b_{t} \bar{S}_{X \cup Y}=0$, so we have

$$
|X| !|Y| ! u b_{t} G_{X, Y}=0 .
$$

Since $U$ is assumed to be free as a $\mathbf{Z}$-module, it follows that $u b_{t} G_{X, Y}=0$, as required.

To show that the homomorphisms $f_{\mathcal{P}}$ are well-defined it suffices to check that $e_{t_{\lambda}} f_{\mathcal{P}}$ satisfies the relations in the previous lemma.

Proposition 1 Let $m \geq 3$ be odd and let $n \in \mathbf{N}$. Suppose that $\mathcal{P}$ is a closed set family of shape $\left(\mathrm{m}^{n}\right)$ and type $\lambda$ and that $u$ is the indexed set partition associated to $\mathcal{P}$. Let $t=t_{\lambda}$. If $X$ and $Y$ are as in the statement of Lemma 1 then

$$
u b_{t} \sum_{\sigma \in S_{X \cup Y}} \sigma \operatorname{sgn}(\sigma)=0 .
$$

Proof Let $\tau \in C(t)$. Suppose that there exist $i_{x} \in X$ and $(i+1)_{y} \in Y$ which appear in the same set in $u \tau$. Then $u \tau\left(1-\left(i_{x},(i+1)_{y}\right)\right)=0$, and, taking coset representatives for $\left\langle\left(i_{x},(i+1)_{y}\right)\right\rangle$ in $S_{X \cup Y}$, we see that $u \tau \sum_{\sigma \in S_{X \cup Y}} \sigma \operatorname{sgn}(\sigma)=0$.

It therefore suffices to show that

$$
u \sum_{\tau \in C^{\prime}} \tau \operatorname{sgn}(\tau) \sum_{\sigma \in S_{X \cup Y}} \sigma \operatorname{sgn}(\sigma)=0
$$

where $C^{\prime}$ is the set of $\tau \in C(t)$ such that no set in $u \tau$ meets both $X$ and $Y$. We may assume that $C^{\prime}$ is non-empty.

Let $\vartheta \in C^{\prime}$ and let $v=u \vartheta$. None of the $|Y|$ sets in $v$ meeting $Y$ can contain an element of $X$. At most $\lambda_{i}^{\prime}-|X|$ of them can contain an element of the complementary set $X^{\prime}=\left\{i_{x^{\prime}}: 1 \leq x^{\prime} \leq \lambda_{i}^{\prime}, i_{x^{\prime}} \notin X\right\}$. By hypothesis $\lambda_{i}^{\prime}-|X|<|Y|$. Hence if there are $s$ sets which meet both $Y$ and $X^{\prime}$ then, after casting out these sets, we are left with at 
least $|Y|-s>|Y|+|X|-\lambda_{i}^{\prime}$ sets which meet $Y$ but not $X^{\prime}$. Since $\mathcal{P}$ is closed, for each such set

$$
B=\left\{c(1)_{b(1)}, c(2)_{b(2)}, \ldots, c(m-1)_{b(m-1)},(i+1)_{y}\right\}
$$

in $v$, there is a corresponding set

$$
A=\left\{c(1)_{a(1)}, c(2)_{a(2)}, \ldots, c(m-1)_{a(m-1)}, i_{z}\right\}
$$

which also appears in $v$. Note that the indices $a(1), \ldots, a(m-1), z$ are determined by the numbers $c(1), \ldots, c(m-1), i$. Since $s$ of the elements of $X^{\prime}$ appear in sets which also meet $Y$, at most $\left|X^{\prime}\right|-s$ of the sets $A$ can have $i_{z} \in X^{\prime}$. Hence at least $(|Y|-s)-\left(\left|X^{\prime}\right|-s\right)=|Y|-\left|X^{\prime}\right|=|Y|+|X|-\lambda_{i}^{\prime}$ of the sets $A$ have $i_{z} \in X$. Therefore we may find sets $B$ and $A$ in $v$ so that

$$
\begin{aligned}
B & =\left\{c(1)_{b(1)}, c(2)_{b(2)}, \ldots, c(m-1)_{b(m-1)},(i+1)_{y}\right\}, \\
A & =\left\{c(1)_{a(1)}, c(2)_{a(2)}, \ldots, c(m-1)_{a(m-1)}, i_{x}\right\},
\end{aligned}
$$

where $(i+1)_{y} \in Y$ and $i_{x} \in X$.

Let

$$
\pi=\left(c(1)_{a(1)}, c(1)_{b(1)}\right) \cdots\left(c(m-1)_{a(m-1)}, c(m-1)_{b(m-1)}\right) .
$$

Since $B\left(i_{x},(i+1)_{y}\right)=A \pi$ we have

$$
v\left(i_{x},(i+1)_{y}\right)=v \pi .
$$

No set in $v \pi$ meets both $X$ and $Y$, so since $v \pi=u \vartheta \pi$, we have $\vartheta \pi \in C^{\prime}$. Thus $u \vartheta$ and $u \vartheta \pi$ are distinct summands of $u \sum_{\tau \in C^{\prime}} \tau \operatorname{sgn}(\tau)$, appearing with the same sign. (This is the only point where we use our hypotheses on $m$.) If $\sigma_{1}, \ldots, \sigma_{s}$ is a set of right-coset representatives for the cosets of $\left\langle\left(i_{x},(i+1)_{y}\right)\right\rangle$ in $S_{X \cup Y}$ then, by (2),

$$
(u \vartheta+u \vartheta \pi) \sum_{\sigma \in S_{X \cup Y}} \sigma \operatorname{sgn}(\sigma)=v(1+\pi)\left(1-\left(i_{x},(i+1)_{y}\right)\right) \sum_{r=1}^{s} \sigma_{r} \operatorname{sgn}\left(\sigma_{r}\right)=0 .
$$

Let $H$ be the subgroup of $C(t)$ of elements that fix all the entries in columns $i$ and $i+1$ of $t$. We have shown that given any $\vartheta \in C^{\prime}$, there exists a non-identity even permutation $\pi_{\vartheta} \in H$ such that $\vartheta \pi_{\vartheta} \in C^{\prime}$ and the contributions to (1) from $u \vartheta$ and $u \vartheta \pi_{\vartheta}$ cancel.

Let $C_{i, i+1}^{\prime}$ be the subset of $C^{\prime}$ of elements which only move entries in columns $i$ and $i+1$ of $t$. Let $\vartheta, \vartheta^{\prime} \in C_{i, i+1}^{\prime}$. If $\vartheta \pi_{\vartheta}=\vartheta^{\prime}$ then $\vartheta^{-1} \vartheta^{\prime}=\pi_{\vartheta}$; since $\pi_{\vartheta}$ fixes the entries in columns $i$ and $i+1$ of $\vartheta$, this implies that $\vartheta^{\prime}=\vartheta$. Hence if the sets $\left\{\vartheta, \vartheta \pi_{\vartheta}\right\},\left\{\vartheta^{\prime}, \vartheta^{\prime} \pi_{\vartheta^{\prime}}\right\}$ meet then $\vartheta=\vartheta^{\prime}$. We may therefore pair up the elements of $C_{i, i+1}^{\prime}$ to show that

$$
u \sum_{\vartheta \in C_{i, i+1}^{\prime}} \vartheta \operatorname{sgn}(\vartheta) \sum_{\sigma \in S_{X \cup Y}} \sigma \operatorname{sgn}(\sigma)=0
$$


There exist $\tau_{1}, \ldots, \tau_{k} \in H$ such that

$$
C^{\prime}=\tau_{1} C_{i, i+1}^{\prime} \cup \cdots \cup \tau_{k} C_{i, i+1}^{\prime}
$$

where the union is disjoint. Hence the left-hand-side of (1) is

$$
\begin{aligned}
& u \sum_{r=1}^{k} \tau_{r} \operatorname{sgn}\left(\tau_{r}\right) \sum_{\vartheta \in C_{i, i+1}^{\prime}} \vartheta \operatorname{sgn}(\vartheta) \sum_{\sigma \in S_{X \cup Y}} \sigma \operatorname{sgn}(\sigma) \\
& \quad=\left(u \sum_{\vartheta \in C_{i, i+1}^{\prime}} \vartheta \operatorname{sgn}(\vartheta) \sum_{\sigma \in S_{X \cup Y}} \sigma \operatorname{sgn}(\sigma)\right) \sum_{r=1}^{k} \tau_{r} \operatorname{sgn}\left(\tau_{r}\right)=0,
\end{aligned}
$$

as we required.

To complete the proof of Theorem 1, we must show that the homomorphisms $f_{\mathcal{P}}$ are injective. This follows from the following general result.

Lemma 2 Let $\lambda$ be a partition of $r$ and let $M$ be a $\mathbf{Z}$-free $\mathbf{Z} S_{r}$-module. If $f: S^{\lambda} \rightarrow M$ is a non-zero homomorphism of $\mathbf{Z} S_{r}$-modules then $f$ is injective.

Proof The homomorphism $f$ induces a non-zero homomorphism

$$
f^{\prime}: S^{\lambda} \otimes_{\mathbf{Z}} \mathbf{Q} \rightarrow M \otimes_{\mathbf{Z}} \mathbf{Q} .
$$

Since $S^{\lambda} \otimes_{\mathbf{Z}} \mathbf{Q}$ is irreducible (see [7, Theorem 4.12]), $f^{\prime}$ is injective. Hence the original map $f$ is also injective.

\section{Minimal constituents of Foulkes characters}

In this section we prove Theorems 2 and 3 on the minimal constituents of the Foulkes characters $\phi^{\left(m^{n}\right)}$.

\subsection{Even case}

Let $m$ be even. To prove part (i) of Theorem 2, we must show that the unique minimal constituent of the Foulkes character $\phi^{\left(m^{n}\right)}$ is $\chi^{\left(m^{n}\right)}$. We do this using properties of the Young permutation modules defined in Sect. 3.1.

Let $q: M^{\left(m^{n}\right)} \rightarrow H^{\left(m^{n}\right)}$ be the map which sends an $\left(m^{n}\right)$-tabloid to the indexed set partition whose member sets are the rows of the tabloid. It is clear that $q$ is a surjective homomorphism of $\mathbf{Z} S_{m n}$-modules. It easily follows that if $\pi^{\left(m^{n}\right)}$ is the character of $M^{\left(m^{n}\right)}$ then

$$
\left\langle\pi^{\left(m^{n}\right)}, \chi^{\lambda}\right\rangle \geq\left\langle\phi^{\left(m^{n}\right)}, \chi^{\lambda}\right\rangle
$$

for every partition $\lambda$ of $m n$. 
The constituents of the character $\pi^{\left(m^{n}\right)}$ are given by Young's rule: see [7, Chap. 14]. Young's rule implies that $\chi^{\left(m^{n}\right)}$ is the unique minimal constituent of $\pi^{\left(m^{n}\right)}$, and that $\left\langle\pi^{\left(m^{n}\right)}, \chi^{\left(m^{n}\right)}\right\rangle=1$. The following lemma implies that there is a nonzero homomorphism $S^{\left(m^{n}\right)} \rightarrow H^{\left(m^{n}\right)}$, and hence $\left\langle\phi^{\left(m^{n}\right)}, \chi^{\left(m^{n}\right)}\right\rangle \geq 1$. Therefore $\chi^{\left(m^{n}\right)}$ is the unique minimal constituent of $\phi^{\left(m^{n}\right)}$ and $\left\langle\phi^{\left(m^{n}\right)}, \chi^{\left(m^{n}\right)}\right\rangle=1$, as required.

Lemma 3 The Specht module $S^{\left(m^{n}\right)}$ is not contained in the kernel of $q$.

Proof Let $t=t_{\left(m^{n}\right)}$ and let $\mathbf{t} \in M^{\left(m^{n}\right)}$ be the corresponding tabloid. By the definition of $q$,

$$
\mathbf{t} q=\left\{\left\{1_{1}, 2_{1}, \ldots, m_{1}\right\}, \ldots,\left\{1_{n}, 2_{n}, \ldots, m_{n}\right\}\right\} .
$$

Let $H \leq C(t)$ be the subgroup of the column permuting group of $t$ that permutes as blocks for its action the rows of $t$. (As an abstract group, $H \cong S_{n}$.) For example, if $m=4$ and $n=3$, then $H$ is generated by $\left(1_{1}, 1_{2}\right)\left(2_{1}, 2_{2}\right)\left(3_{1}, 3_{2}\right)\left(4_{1}, 4_{2}\right)$ and $\left(1_{1}, 1_{2}, 1_{3}\right)\left(2_{1}, 2_{2}, 2_{3}\right)\left(3_{1}, 3_{2}, 3_{3}\right)\left(4_{1}, 4_{2}, 4_{3}\right)$.

Note that since $m$ is even, every element of $H$ is an even permutation. Let $K \leq$ $C(t)$ be the subgroup of permutations which fix the elements $1_{1}, \ldots, 1_{n}$ in the first column of $t$. It is easy to see that $C(t)=H K$, and so

$$
e_{t} q=(\mathbf{t} q) \sum_{\tau \in C(t)} \tau \operatorname{sgn}(\tau)=(\mathbf{t} q) \sum_{\pi \in H} \pi \sum_{\sigma \in K} \sigma \operatorname{sgn}(\sigma)=n !(\mathbf{t} q) \sum_{\sigma \in K} \sigma \operatorname{sgn}(\sigma) .
$$

The summands on the right-hand-side are distinct basis elements of $H^{\left(m^{n}\right)}$, hence $e_{t} q \neq 0$.

\subsection{Odd case}

We start with the following general form for a homomorphism from a Specht module into a Foulkes module $H^{\left(m^{n}\right)}$ when $m$ is odd.

Proposition 2 Let $m$ be odd and let $n \in \mathbf{N}$. Let $\lambda$ be a partition of $m n$ and let $f: S^{\lambda} \rightarrow H^{\left(m^{n}\right)}$ be a homomorphism of $\mathbf{Z} S_{m n}$-modules. Let $t=t_{\lambda}$. There exist set families $\mathcal{P}_{1}, \ldots, \mathcal{P}_{k}$ of shape $\left(m^{n}\right)$ and type $\lambda$ and integers $a_{1}, \ldots, a_{k}$ such that

$$
e_{t} f=a_{1} u_{1} b_{t}+\cdots+a_{k} u_{k} b_{t}
$$

where $u_{i}$ is the indexed set partition associated to $\mathcal{P}_{i}$.

Proof Let

$$
e_{t} f=\sum_{u} c_{u} u
$$

where the sum is over all indexed set partitions $u$ of shape $\left(m^{n}\right)$ and type $\lambda$ and $c_{u} \in \mathbf{Z}$. 
Let $u$ be such an indexed set partition. If the symbols $i_{j}$ and $i_{k}$ appear in the same set in $u$ then, since $e_{t}\left(i_{j}, i_{k}\right)=-e_{t}$ and $u\left(i_{j}, i_{k}\right)=u$, we must have $c_{u}=0$. Now suppose that $u$ contains two sets

$$
\begin{aligned}
& \left\{c(1)_{a(1)}, c(2)_{a(2)}, \ldots, c(m)_{a(m)}\right\} \\
& \left\{c(1)_{b(1)}, c(2)_{b(2)}, \ldots, c(m)_{b(m)}\right\}
\end{aligned}
$$

which become equal if the indices are removed. Let

$$
\tau=\left(c(1)_{a(1)}, c(1)_{b(1)}\right) \cdots\left(c(m)_{a(m)}, c(m)_{b(m)}\right) .
$$

Since $m$ is odd, $\tau$ is an odd permutation in $C(t)$. Hence $e_{t} \tau=-e_{t}$ and $u \tau=u$, and again we have $c_{u}=0$.

These remarks show that if $c_{u} \neq 0$ then removing indices from the symbols in the sets making up $u$ leaves a set family of shape $\left(\mathrm{m}^{n}\right)$ and type $\lambda$. If removing indices from $u$ and $v$ gives the same set family then $v=u \tau$ for some $\tau \in C(t)$. Since $e_{t} \tau=\operatorname{sgn}(\tau) e_{t}$ we have $c_{v}=\operatorname{sgn}(\tau) c_{u}$. The proposition follows.

We also need a corollary of the following combinatorial proposition, which will be used again in Sect. 6 below.

Proposition 3 If $\mathcal{P}$ is a minimal set family then $\mathcal{P}$ is closed.

Proof It will be necessary in this proof to extend the definition of type so that it applies to all set families. We define the conjugate type of a set family $\mathcal{P}$ of shape $\left(m^{n}\right)$ to be the composition $\alpha$ such that $\alpha_{i}$ is the number of sets in $\mathcal{P}$ containing $i$. Note that if $\alpha$ is a partition, then $\alpha^{\prime}$ is the type (in the usual sense) of $\mathcal{P}$.

Let $\mathcal{P}$ be a set family of shape $\left(m^{n}\right)$ and type (in the usual sense) $\lambda$. Suppose that $\mathcal{P}$ is not closed. We may find $A \in \mathcal{P}$ and $i \in \mathbf{N}$ such that $i+1 \in A$ and the set

$$
B=A \backslash\{i+1\} \cup\{i\}
$$

is not in $\mathcal{P}$. Let $\mathcal{Q}$ be the set family obtained from $\mathcal{P}$ by removing $A$ and adding $B$. If $\alpha=\lambda^{\prime}$, then the conjugate type of $\mathcal{Q}$ is $\beta$ where $\beta_{i}=\alpha_{i}+1, \beta_{i+1}=\alpha_{i+1}-1$ and $\beta_{j}=\alpha_{j}$ if $j \neq i, i+1$. Hence $\beta \triangleright \alpha$ (where $\triangleright$ now refers to the dominance order on compositions). Iterating this construction, we will reach a closed set family $\mathcal{R}$ of conjugate type $\gamma$ where $\gamma \triangleright \alpha$. Since $\mathcal{R}$ is closed, $\gamma$ is a partition. If $v=\gamma^{\prime}$ then, $v^{\prime} \triangleright \lambda^{\prime}$, and so $v \triangleleft \lambda$. Thus $\mathcal{R}$ has smaller type than $\mathcal{P}$ and so $\mathcal{P}$ is not minimal.

Corollary 1 If $\left\langle\phi^{\left(m^{n}\right)}, \chi^{\lambda}\right\rangle \geq 1$ then there is a minimal set family of shape $\left(m^{n}\right)$ and type $\mu$ where $\mu \unlhd \lambda$, and this set family is closed.

Proof The hypothesis implies that there is an injective homomorphism of $\mathbf{Q} S_{m n^{-}}$ modules

$$
f: S^{\lambda} \otimes_{\mathbf{Z}} \mathbf{Q} \rightarrow H^{\left(m^{n}\right)} \otimes_{\mathbf{Z}} \mathbf{Q}
$$


Let

$$
e_{t_{\lambda}} f=\sum_{u} c_{u} u
$$

where $c_{u} \in \mathbf{Q}$ and the sum is over all indexed set partitions $u$ of shape $\left(m^{n}\right)$ and type $\lambda$. For each such $u$, let $c_{u}=a_{u} / b_{u}$ where $a_{u}, b_{u} \in \mathbf{Z}$. Let $m$ be the product of all the $b_{u}$. It is easy to see that the map $g: S^{\lambda} \rightarrow H^{\left(m^{n}\right)}$ defined by

$$
e_{t_{\lambda}} g=\sum_{u} m c_{u} u
$$

is a well-defined injective homomorphism of $\mathbf{Z} S_{m n}$-modules.

Applying Proposition 2 to $g$, we see that there is a set family $\mathcal{P}$ of shape $\left(m^{n}\right)$ and type $\lambda$. Proposition 3 implies that if $\mathcal{Q}$ is a set family of minimal type $\mu \unlhd \lambda$ then $\mathcal{Q}$ is closed.

We are now ready to prove part (ii) of Theorem 2. Suppose that $\chi^{\lambda}$ is a minimal constituent of $\phi^{\left(m^{n}\right)}$. By Corollary 1 there is a minimal set family $\mathcal{Q}$ of type $\mu$ where $\mu \unlhd \lambda$. Since $\mathcal{Q}$ is closed, it follows from Theorem 1 that there is a non-zero homomorphism $S^{\mu} \rightarrow H^{\left(m^{n}\right)}$, and so $\left\langle\phi^{\left(m^{n}\right)}, \chi^{\mu}\right\rangle \geq 1$. Therefore $\mu=\lambda$ and $\mathcal{Q}$ is a minimal set family of type $\lambda$.

Conversely, suppose that there is a minimal set family $\mathcal{P}$ of type $\lambda$. By Proposition 3, $\mathcal{P}$ is closed, and so it follows from Theorem 1 that $\chi^{\lambda}$ is a summand of $\phi^{\left(m^{n}\right)}$. If there is a summand $\chi^{\mu}$ of $\phi^{\left(m^{n}\right)}$ with $\mu \triangleleft \lambda$, then by Proposition 2 , there is a set family of type $\mu$; this contradicts the minimality of $\mathcal{P}$. Therefore $\chi^{\lambda}$ is a minimal constituent of $\phi^{\left(m^{n}\right)}$.

\subsection{Proof of Theorem 3}

Suppose that $\chi^{\lambda}$ is a minimal constituent of $\phi^{\left(m^{n}\right)}$. By Theorem $2, \lambda$ is the type of a minimal set family of shape $\left(m^{n}\right)$. Let $u_{1}, \ldots, u_{d}$ be the indexed set partitions associated to the set families $\mathcal{P}_{1}, \ldots, \mathcal{P}_{d}$ of shape $\left(m^{n}\right)$ and type $\lambda$. Proposition 3 implies that the $\mathcal{P}_{r}$ are closed. By Proposition 2 we know that if $f: S^{\lambda} \rightarrow H^{\left(m^{n}\right)}$ is a homomorphism of $\mathbf{Z} S_{m n}$-modules, then there exist $a_{1}, \ldots, a_{d} \in \mathbf{Z}$ such that

$$
e_{t} f=a_{1} u_{1} b_{t}+\cdots+a_{d} u_{d} b_{t}
$$

where $t=t_{\lambda}$. Hence

$$
f=a_{1} f_{\mathcal{P}_{1}}+\cdots+a_{d} f_{\mathcal{P}_{d}} .
$$

To show that homomorphisms $f_{\mathcal{P}_{1}}, \ldots, f_{\mathcal{P}_{d}}$ are linearly independent it suffices to show that the images of $e_{t}$,

$$
e_{t} f_{\mathcal{P}_{r}}=\sum_{\tau \in C(t)} u_{r} \tau \operatorname{sgn}(\tau)
$$

are linearly independent. Given $\tau \in C(t)$ and $r \in\{1, \ldots, d\}$, we can easily recover $\mathcal{P}_{r}$ from $u_{r} \tau$ by removing the indices from the symbols in the sets making up $u_{r} \tau$. Therefore each $e_{t} f_{\mathcal{P}_{r}}$ is a sum of different basis elements of $H^{\left(m^{n}\right)}$; as such, they are linearly independent. 


\section{Set families and partitions}

\subsection{Minimal and unique set families}

We proved in Proposition 3 that minimal set families are closed. The following proposition implies that if $\mathcal{P}$ is the unique set family of its shape and type, then $\mathcal{P}$ is minimal. This establishes the following chain of implications on set families of a given shape:

$$
\text { unique of its type } \Longrightarrow \text { minimal } \Longrightarrow \text { closed. }
$$

Proposition 4 If $\mathcal{P}$ is a set family of shape $\left(m^{n}\right)$ and type $\lambda$ and $\mu \triangleright \lambda$ then there are two distinct set families of shape $\left(m^{n}\right)$ and type $\mu$.

Proof We may assume that $\lambda$ and $\mu$ are neighbours in the dominance order, so $\mu$ is obtained from $\lambda$ by moving a box upwards in its Young diagram. Suppose the box is moved from column $i$ to column $j>i$. We have $\mu_{i}^{\prime}=\lambda_{i}^{\prime}-1, \mu_{j}^{\prime}=\lambda_{j}^{\prime}+1$ and $\mu_{k}^{\prime}=\lambda_{k}^{\prime}$ if $k \neq i, j$.

The sets in $\mathcal{P}$ either contain both $i$ and $j$, or $i$ alone, or $j$ alone, or neither. Since $\lambda_{i}^{\prime}-\lambda_{j}^{\prime}=\mu_{i}^{\prime}-\mu_{j}^{\prime}+2$, at least two more sets contain $i$ alone than contain $j$ alone. Hence there are two sets

$$
\begin{aligned}
A & =\{x(1), x(2), \ldots, x(m-1), i\} \in \mathcal{P}, \\
A^{\prime} & =\left\{x^{\prime}(1), x^{\prime}(2), \ldots, x^{\prime}(m-1), i\right\} \in \mathcal{P}
\end{aligned}
$$

such that

$$
\begin{aligned}
B & =\{x(1), x(2), \ldots, x(m-1), j\} \notin \mathcal{P}, \\
B^{\prime} & =\left\{x^{\prime}(1), x^{\prime}(2), \ldots, x^{\prime}(m-1), j\right\} \notin \mathcal{P} .
\end{aligned}
$$

Let $\mathcal{Q}$ be the set family obtained from $\mathcal{P}$ by removing $A$ and adding $B$, and let $\mathcal{Q}^{\prime}$ be the set family obtained from $\mathcal{P}$ by removing $A^{\prime}$ and adding $B^{\prime}$. Then $\mathcal{Q}$ and $\mathcal{Q}^{\prime}$ are two different set families of type $\mu$.

To complete the proof of Theorem 4, we must show that neither of the implications in the chain (3) is reversible. We shall use the following definition throughout the remainder of this section.

Definition 8 Let $A$ be a subset of the natural numbers. The downset of $A$, denoted $A \preceq$, is the set family consisting of all subsets $X$ such that $X \preceq A$.

It is obvious that a set family is closed if and only if it is a union of downsets.

Our first example concerns the downset $\mathcal{P}=\{2,4,6,8\} \preceq$. This is a closed set family of shape $\left(4^{42}\right)$, but it is not unique for its type, since the set family

$$
Q=\mathcal{P} \backslash\{\{2,4,6,8\},\{1,3,5,7\}\} \cup\{\{1,2,7,8\},\{3,4,5,6\}\}
$$


Fig. 1 Construction of the partition $\left(5^{8}\right) \star(4,2,1)$

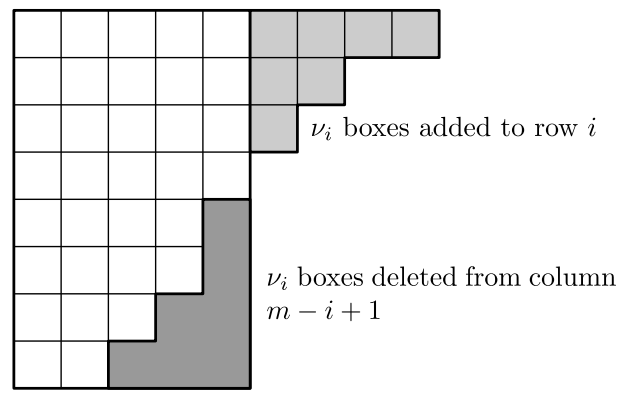

has the same shape and type as $\mathcal{P}$. Neither is minimal. This is most easily seen by noting that since $\mathcal{Q}$ contains $\{1,3,5,8\}$ but not $\{1,3,5,7\}, \mathcal{Q}$ is not closed. Following the proof of Proposition 3 leads one to (amongst others) the closed set family

$$
\begin{aligned}
R & =\mathcal{Q} \backslash\{\{2,4,5,8\}\} \cup\{\{1,3,5,7\}\} \\
& =\mathcal{P} \backslash\{\{2,4,6,8\},\{2,4,5,8\}\} \cup\{\{1,2,7,8\},\{3,4,5,6\}\} \\
& =\{1,2,7,8\} \preceq \cup\{1,4,6,8\} \preceq \cup\{2,3,6,8\} \preceq \cup\{2,4,6,7\} \preceq \cup\{3,4,5,6\} \preceq
\end{aligned}
$$

which has smaller type than $\mathcal{P}$ and $\mathcal{Q}$. (In fact, $\mathcal{R}$ is minimal.)

The following example of a minimal set family that is not unique for its type was found by a computer search for minimal set families with prescribed shape and maximum entry. (A description of the algorithm used and accompanying source code is available from the second author's website, www.ma.rhul.ac.uk/ uvah099/.) Let

$$
\begin{aligned}
\mathcal{S}= & \{1,5,9\}^{\preceq} \cup\{1,6,8\}^{\preceq} \cup\{2,6,7\}^{\preceq} \cup\{3,4,8\}^{\preceq} \cup\{3,5,6\}^{\preceq}, \\
\mathcal{S}^{\prime}= & \{1,4,9\}^{\preceq} \cup\{1,7,8\}^{\preceq} \cup\{2,3,9\}^{\preceq} \cup\{2,4,8\}^{\preceq} \\
& \cup\{2,5,7\}^{\preceq} \cup\{3,4,7\}^{\preceq} \cup\{4,5,6\}^{\preceq} .
\end{aligned}
$$

The set families $\mathcal{S}$ and $\mathcal{S}^{\prime}$ both have type $\lambda=(24,19,17,16,13,12,10,8,4)^{\prime}$ and shape $\left(3^{41}\right)$. Using the computer to enumerate all closed set families of shape $\left(3^{41}\right)$ with maximum entry $\leq 9$ confirms that there are no set families of shape $\left(3^{41}\right)$ with type $\triangleleft \lambda$.

\subsection{Constructing types of minimal set families}

Let $m, n \in \mathbf{N}$. Given a partition $v$ of $n-1$ with $k \leq m$ parts, we let $\left(m^{n}\right) \star v$ denote the partition obtained from $\left(m^{n}\right)$ by deleting $v_{i}$ boxes from column $m+1-i$ and then adding $v_{i}$ boxes to row $i$, for each $i$ such that $1 \leq i \leq k$. This construction is illustrated in Fig. 1. (If $v_{1}=a$ then we add boxes to rows $1,2, \ldots, k$, and remove boxes from rows $n-a+1, \ldots, n$; since $k+a \leq n$, the partition $\left(m^{n}\right) \star v$ is welldefined.)

Our first object in this section is to prove the following proposition.

Proposition 5 Let $v$ be a partition of $n-1$ with at most $m$ parts. There is a unique set family of shape $\left(m^{n}\right)$ and type $\left(m^{n}\right) \star v$. 
Proof Suppose that $v$ has exactly $k$ parts. Let $A=\{1,2, \ldots, m\}$. It is easily checked that

$$
\mathcal{P}=\{A\} \cup\left\{A \backslash\{m-i+1\} \cup\{m+j\}: 1 \leq i \leq k, 1 \leq j \leq v_{i}\right\}
$$

is a set family of shape $\left(m^{n}\right)$ and type $\left(m^{n}\right) \star v$.

Now suppose that $\mathcal{Q}$ is a set family of this shape and type. We may write

$$
\mathcal{Q}=\left\{A \backslash B_{r} \cup C_{r}: 1 \leq r \leq n\right\}
$$

for some subsets $B_{r}, C_{r} \subseteq \mathbf{N}$ such that $B_{r} \cap C_{r}=\varnothing$ for $1 \leq r \leq n$. For each $i$ such that $1 \leq i \leq k$, exactly $\nu_{i}$ of the sets $B_{1}, \ldots, B_{n}$ contain $m-i+1$. It follows that $\left|B_{1}\right|+\cdots+\left|B_{n}\right|=n-1$, and so one of the sets $B_{r}$ is empty, and the remaining $n-1$ are singletons. Hence $A \in \mathcal{Q}$, and for each $i$ such that $1 \leq i \leq k$, there are exactly $v_{i}$ sets in $\mathcal{Q}$ of the form $A \backslash\{m-i+1\} \cup\{c\}$ where $c \in\left\{m+1, \ldots, m+v_{1}\right\}$. Looking first at the case $i=1$, we see that $A \backslash\{m\} \cup\{m+j\} \in \mathcal{Q}$ for each $j$ such that $1 \leq j \leq v_{1}$. Iterating this argument for $i=2, \ldots, k$ shows that $\mathcal{Q}=\mathcal{P}$.

We isolate the following corollary of Proposition 5.

Corollary 2 Let $m$ be odd and let $n \in \mathbf{N}$. If $v$ is a partition of $n-1$ with at most $m$ parts then

$$
\left\langle\phi^{\left(m^{n}\right)}, \chi^{\left(m^{n}\right) \star v}\right\rangle=1 .
$$

Moreover $\left\langle\phi^{\left(m^{n}\right)}, \chi^{\mu}\right\rangle=0$ if $\mu \triangleleft \chi^{\left(m^{n}\right) \star \nu}$.

Proof By Theorem 4, $\left(m^{n}\right) \star v$ is the type of a minimal set family of shape $\left(m^{n}\right)$. The result now follows from Theorem 3 .

It is natural to ask when every minimal constituent $\phi^{\left(m^{n}\right)}$ arises from this construction. We shall show in Proposition 6 below that this is the case if and only if $n \leq 5$ or $m=1$. We begin with the following straightforward lemma.

Lemma 4 Let $\mathcal{P}$ be a closed set family of shape $\left(m^{n}\right)$ where $m \geq n$. If $X \in \mathcal{P}$ then $X \supseteq\{1,2, \ldots, m-n+1\}$.

Proof Suppose that the smallest number not present in $X$ is $m-t+1$, so $X=$ $\{1,2, \ldots, m-t, x(1), \ldots, x(t)\}$ for some $x(r)>m-t+1$. For each $r$ such that $1 \leq r \leq t$, the set $X \backslash\{x(r)\} \cup\{m-t+1\}$ is majorized by $X$, so must lie in $\mathcal{P}$. Hence $|\mathcal{P}| \geq t+1$, and so $t \leq n-1$. It follows that $m-t \geq m-n+1$, as required.

In the proof of the following lemma, a further construction on partitions will be found useful: given a partition $\lambda$ with exactly $k$ parts, each of size $\geq c$, let $\lambda-\left(c^{k}\right)$ denote the partition obtained from $\left(\lambda_{1}-c, \ldots, \lambda_{k}-c\right)$ by removing any final parts of size zero.

Lemma 5 Let $m \in \mathbf{N}$ and let $n \leq 5$. If $\mathcal{P}$ is a closed set family of shape $\left(m^{n}\right)$ then $\mathcal{P}$ has type $\left(m^{n}\right) \star v$ for some partition $v$ of $n-1$ with at most $m$ parts. 


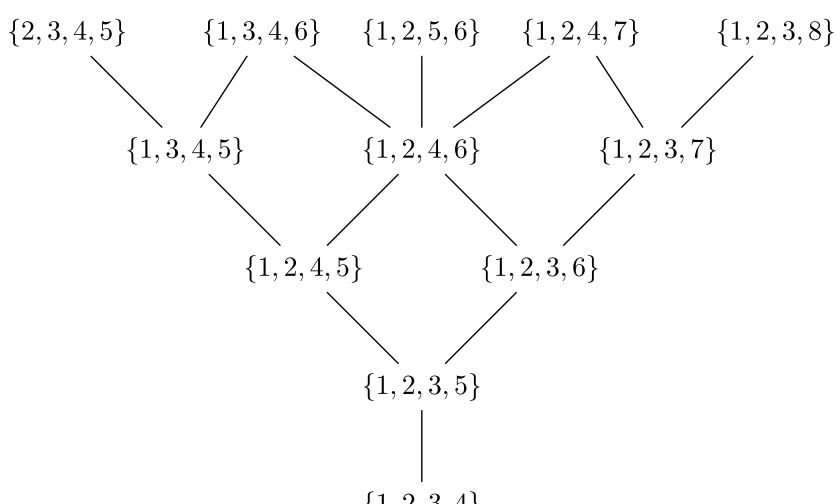

$\{1,2,3,4\}$

Fig. 2 The first five levels of the lattice of 4-subsets of $\mathbf{N}$ under the majorization order

Proof If $m<n$ then the result can be checked directly. For example, when $m=4$ and $n=5$, the set family $\mathcal{P}$ must consist of 5 sets taken from the first 5 levels of the lattice of 4 -subsets of $\mathbf{N}$ under the majorization order. It is easily seen from Fig. 2 that there are 5 possibilities for $\mathcal{P}$, namely $\{1,2,3,8\} \preceq,\{1,2,3,7\} \preceq \cup\{\{1,2,4,5\}\}$, $\{1,2,4,6\} \preceq,\{1,3,4,5\} \preceq \cup\{\{1,2,3,6\}\}$ and $\{2,3,4,5\} \preceq$. The types of these set families are $\left(4^{5}\right) \star v$ where $v=(4),(3,1),(2,2),(2,1,1)$ and $\left(1^{4}\right)$, respectively.

Now suppose that $m \geq n$. By Lemma 4 , we know that every set in $\mathcal{P}$ contains $\{1,2, \ldots, m-n+1\}$. Let $\mathcal{Q}$ be the set family obtained by removing the elements $1,2, \ldots, m-n+1$ from every set in $\mathcal{P}$, and then subtracting $m-n+1$ from each remaining element. The shape of $\mathcal{Q}$ is $\left((n-1)^{n}\right)$. If $\mathcal{P}$ has type $\lambda$, where $\lambda$ has exactly $k$ parts, then $\mathcal{Q}$ has type $\lambda-\left((m-n+1)^{k}\right)$. By the result already proved, we know that

$$
\lambda-\left((m-n+1)^{k}\right)=\left((n-1)^{n}\right) \star v
$$

for some partition $v$ of $n-1$. Since $\lambda-\left((m-n+1)^{k}\right)$ is a partition of $(n-1) k$, we must have $k=n$. It is now easy to see that $\lambda=\left(m^{n}\right) \star v$, as required.

We now show that Lemma 5 is false when $n \geq 6$ and $m \geq 2$.

Lemma 6 Let $n \geq 6$ and let $m \geq 2$. The unique set family of shape $\left(m^{n}\right)$ and type $\left(m+n-4, m+2, m+2,(m-1)^{n-6},(m-2)^{3}\right)$ is

$$
\{1,2, \ldots, m-2, m+1, m+2\} \preceq \cup\{1,2, \ldots, m-2, m-1, m+n-4\} \preceq .
$$

This type is not of the form $\left(m^{n}\right) \star v$ for any partition $v$ of $n-1$ with at most $m$ parts.

Proof It is routine to check that this set family does have the claimed type. Suppose that $\mathcal{P}$ is a set family of this type. Every set in $\mathcal{P}$ contains $\{1,2, \ldots, m-2\}$, so, as in the proof of Lemma 5, we may pass to a set family $\mathcal{Q}$ of shape $\left(2^{n}\right)$ and type $\left(n-2,4,4,1^{n-6}\right)$ by removing the elements $1,2, \ldots, m-2$ from every set, and then subtracting $m-2$ from every remaining element. It suffices to show that $\mathcal{Q}=$ 
$\{3,4\} \preceq \cup\{1, n-2\} \preceq$. Of the $n-3$ sets in $\mathcal{Q}$ which contain 1 , at most one can contain 2 , so 2 must appear in two of the three remaining sets in $\mathcal{Q}$. A similar argument with 3 and 4 shows that $\{2,3\},\{2,4\},\{3,4\} \in \mathcal{Q}$. It is now clear that $\mathcal{Q}$ is as claimed.

If the final assertion in the lemma is false, then the type of $Q$ is $\left(2^{n}\right) \star v$ where $v$ is a partition of $n-1$ with at most 2 parts. However, $\left(n-2,4,4,1^{n-6}\right)$ has three parts of size $>2$, so this is impossible.

We now use Lemmas 5 and 6 to prove the following proposition.

Proposition 6 Let $m$ be odd and let $n \in \mathbf{N}$. Every minimal constituent of $\phi^{\left(m^{n}\right)}$ is of the form $\chi^{\left(m^{n}\right) \star v}$ for some partition $v$ of $n-1$ with at most $m$ parts if and only if $m=1$ or $n \leq 5$.

Proof Since $\phi^{\left(1^{n}\right)}=\chi^{(n)}$ and $(n)=\left(1^{n}\right) \star(n-1)$, the proposition holds when $m=1$. When $n \leq 5$ it follows from Lemma 5, Theorem 2 and Theorem 4 . If $n \geq 6$ then, since the unique set family constructed in Lemma 6 is minimal by Theorem 4 , there is a minimal constituent of $\phi^{\left(m^{n}\right)}$ not of the form $\chi^{\left(m^{n}\right) \star v}$ for any partition $v$ of $n-1$.

\section{Minimal constituents of generalised Foulkes characters}

We end by showing how to construct the minimal constituents of a wider class of permutation characters. Let $\mu$ be a partition of $N$ with largest part of size $a$. If $\mu$ has exactly $n(i)$ parts of length $i$ for each $i$ such that $1 \leq i \leq a$, we define the generalised Foulkes character $\phi^{\mu}$ to be the induced character

$$
\phi^{\mu}=\left(\phi^{\left(1^{n(1)}\right)} \times \phi^{\left(2^{n(2)}\right)} \times \cdots \times \phi^{\left(a^{n(a)}\right)}\right) \uparrow_{S_{n(1)} \times S_{2 n(2)} \times \cdots \times S_{a n(a)}} .
$$

If $\mu=\left(m^{n}\right)$ for some $m, n \in \mathbf{N}$ then this definition agrees with the one given earlier.

Our main aim in this section is to prove Proposition 7 below describing the minimal constituents of generalised Foulkes characters. To state this result we need one final construction on partitions.

Definition 9 Given $\lambda$ a partition of $r$ and $\mu$ a partition of $s$, we denote by $\lambda \cup \mu$ the partition of $r+s$ whose multiset of parts is the union of the multisets of parts of $\lambda$ and $\mu$.

For example, $(5,2) \cup(3,2,2,1,1)=(5,3,2,2,2,1,1)$.

Proposition 7 Let $\mu=\left(m(1)^{n(1)}\right) \cup \cdots \cup\left(m(t)^{n(t)}\right)$ where the $m(k)$ are distinct. If $\chi^{\lambda}$ is a minimal constituent of $\phi^{\mu}$ then

$$
\lambda=v(1) \cup v(2) \cup \cdots \cup v(t)
$$

where $\nu(k)$ is a partition of $m(k) n(k)$ and $\chi^{\nu(k)}$ is a minimal constituent of $\phi^{\left(m(k)^{n(k)}\right)}$ for each $k$ such that $1 \leq k \leq t$. 
Fig. 3 Use of the

Littlewood-Richardson rule to show that

$\left(\chi^{(6,3,2)} \times \chi^{(4,3,1)}\right) \uparrow_{S_{10} \times S_{8}}^{S_{18}}$

has

$\chi^{(6,3,2) \cup(4,3,1)}=\chi^{(6,4,3,3,2,1)}$

as a summand

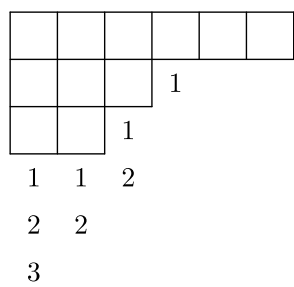

Our proof of Proposition 7 uses the following two general lemmas.

Lemma 7 Let $\lambda$ be a partition of $r$ and let $\mu$ be a partition of $s$. The unique minimal constituent of $\left(\chi^{\lambda} \times \chi^{\mu}\right){ }_{S_{r} \times S_{s}}^{S_{r+s}}$ is $\chi^{\lambda \cup \mu}$.

Proof Let $\vartheta=\left(\chi^{\lambda} \times \chi^{\mu}\right) \uparrow{ }_{S_{r} \times S_{s}}^{S_{r+s}}$. It follows from the description of the LittlewoodRichardson rule given in [7, Chap. 16] that $\chi^{\lambda \cup \mu}$ is a constituent of the character $\vartheta$. A typical example, which shows how the parts of $\lambda \cup \mu$ may be obtained by adding numbers to $\lambda$, is given in Fig. 3. Note that at step $j$, the lowest $\mu_{j}$ positions that are eligible to be filled receive a $j$. For an explanation of the notation and method used, the reader is referred to [7, Chap. 16].

The remainder of the proof can be completed using the easier Young's rule. The character

$$
\psi=\left(\chi^{\lambda} \times\left(1_{S_{\mu} \uparrow} S_{s}\right)\right) \uparrow_{S_{r} \times S_{s}}^{S_{r+s}}
$$

certainly contains all the constituents of $\vartheta$, so to prove the lemma, it suffices to show that $\psi$ has $\chi^{\lambda \cup \mu}$ as its least constituent. This follows by induction on the number of parts of $\mu$ if we rewrite $\psi$ as

$$
\left(\chi^{\lambda} \times 1_{\mu_{1}} \times \cdots \times 1_{\mu_{k}}\right) \uparrow_{S_{r} \times S_{\mu_{1}} \times \cdots \times S_{\mu_{k}}}^{S_{r+s}}
$$

and then repeatedly apply Young's rule (see [7, Chap. 14]).

Lemma 8 Let $\pi$ be a character of $S_{r}$ and let $\vartheta$ be a character of $S_{s}$. If $\chi^{\nu}$ is a minimal constituent of $(\pi \times \vartheta) \uparrow{ }_{S_{r} \times S_{s}}^{S_{r+s}}$ then $v=\lambda \cup \mu$ where $\chi^{\lambda}$ is a minimal constituent of $\pi$ and $\chi^{\mu}$ is a minimal constituent of $\vartheta$.

Proof Let $\psi=(\pi \times \vartheta) \uparrow \stackrel{S_{r+s}}{S_{r} \times S_{s}}$. It follows from Lemma 7 that if $\chi^{v}$ is a minimal constituent of $\psi$ then there are partitions $\lambda$ and $\mu$ such that $\left\langle\pi, \chi^{\lambda}\right\rangle \geq 1,\left\langle\vartheta, \chi^{\mu}\right\rangle \geq 1$ and $v=\lambda \cup \mu$.

Suppose that $\chi^{\lambda}$ is not a minimal constituent of $\pi$. Then there exists a partition $\lambda^{\star}$ such that $\lambda^{\star} \triangleleft \lambda$ and $\left\langle\pi, \chi^{\lambda^{\star}}\right\rangle \geq 1$. By Lemma 7 we have

$$
\left\langle\psi, \chi^{\lambda^{\star} \cup \mu}\right\rangle \geq 1
$$

It is easily seen that $\lambda^{\star} \cup \mu \triangleleft \lambda \cup \mu$; this contradicts the minimality of $\chi^{\nu}$. Therefore $\chi^{\lambda}$ is a minimal constituent of $\pi$ and similarly, $\chi^{\mu}$ is a minimal constituent of $\vartheta$. 
We are now ready to prove Proposition 7. Let $N=m(1) n(1)+\cdots+m(t) n(t)$. Since the $m(r)$ are distinct,

$$
\phi^{\mu}=\left(\phi^{\left(m(1)^{n(1)}\right)} \times \cdots \times \phi^{\left(m(t)^{n(t)}\right)}\right) \uparrow_{S_{m(1) n(1)} \times \cdots \times S_{m(t) n(t)}}^{S_{N}} .
$$

The proposition now follows by repeated applications of Lemma 8.

We finish with the observation that the converse to Proposition 7 (and to Lemma 8) is false. This can be demonstrated using Corollary 2 in Sect. 6.2. It follows from this corollary that $\phi^{\left(5^{5}\right)}$ has

$$
\chi^{\left(5^{5}\right) \star(2,1,1)}=\chi^{(7,6,6,4,2)} \quad \text { and } \quad \chi^{\left(5^{5}\right) \star\left(1^{4}\right)}=\chi^{(6,6,6,6,1)}
$$

as minimal constituents. Similarly, $\phi^{\left(3^{5}\right)}$ has

$$
\chi^{\left(3^{5}\right) \star(4)}=\chi^{(7,2,2,2,2)} \quad \text { and } \quad \chi^{\left(3^{5}\right) \star(3,1)}=\chi^{(6,4,2,2,1)}
$$

as minimal constituents. It is clear that

$$
(6,6,6,6,1) \cup(7,2,2,2,2) \triangleright(7,6,6,4,2) \cup(6,4,2,2,1) .
$$

Hence $\chi^{(6,6,6,6,1) \cup(7,2,2,2,2)}$ is not a minimal constituent of $\phi^{\left(5^{5}, 3^{5}\right)}$, even though it arises from the $\cup$-construction applied to a minimal constituent of $\phi^{\left(5^{5}\right)}$ and a minimal constituent of $\phi^{\left(3^{5}\right)}$.

Acknowledgements The authors thank Johannes Siemons for a helpful conversation. They would also like to thank the anonymous referee for his or her helpful comments on an earlier version of this paper.

\section{References}

1. Brion, M.: Stable properties of plethysm: on two conjectures of Foulkes. Manuscr. Math. 80, 347-371 (1993)

2. Curtis, C.W., Reiner, I.: Representation Theory of Finite Groups and Associative Algebras. AMS Chelsea, Providence (2006). Reprint of the 1962 original

3. Dent, S.C., Siemons, J.: On a conjecture of Foulkes. J. Algebra 226(1), 236-249 (2000)

4. Foulkes, H.O.: Concomitants of the quintic and sextic up to degree four in the coefficients of the ground form. J. Lond. Math. Soc. 25, 205-209 (1950)

5. Fulton, W.: Young Tableaux. London Mathematical Society Student Texts, vol. 35. CUP, Cambridge (1997)

6. Howe, R.: $\left(\mathrm{GL}_{n}, \mathrm{GL}_{m}\right)$-duality and symmetric plethysm. Proc. Indian Acad. Sci. Math. Sci. 97, 85109 (1987)

7. James, G.D.: The representation theory of the symmetric groups. Lecture Notes in Mathematics, vol. 682. Springer, Berlin (1978)

8. McKay, T.: On plethysm conjectures of Stanley and Foulkes. J. Algebra 319(5), 2050-2071 (2008)

9. Müller, J., Neunhöffer, M.: Some computations regarding Foulkes' conjecture. Exp. Math. 14(3), 277-283 (2005)

10. Stanley, R.P.: Positivity problems and conjectures in algebraic combinatorics. In: Mathematics: Frontiers and Perspectives, pp. 295-319. AMS, Providence (2000)

11. Thrall, R.M.: On symmetrized Kronecker powers and the structure of the free Lie ring. Am. J. Math. 64(2), 371-388 (1942) 